\title{
Encapsulation of ethylene gas into granular cold-water-soluble starch: structure and release kinetics
}

\begin{abstract}
Ethylene gas was introduced into granular cold-water-soluble (GCWS) starches using a solid encapsulation method. The morphological and structural properties of the novel inclusion complexes (ICs) were characterized using scanning electron microscopy, X-ray diffractometry, and Raman spectroscopy. The V-type single helix of GCWS starches was formed through controlled gelatinization and ethanol precipitation and was approved to host ethylene gas. The controlled release characteristics of ICs were also investigated at various temperature and relative humidity conditions. Avrami's equation was fitted to understand the release kinetics and showed that the release of ethylene from the ICs was accelerated by increasing temperature or $\mathrm{RH}$ and was decelerated by increased degree of amylose polymerization. The IC of Hylon-7 had the highest ethylene concentration $(31.8 \%, \mathrm{w} / \mathrm{w})$ among the five starches, and the IC of normal potato starch showed the best controlled release characteristics. As a renewable and inexpensive material, GCWS starch is a desirable solid encapsulation matrix with potential in agricultural and food applications.
\end{abstract}

Keyword: Controlled release; Encapsulation; Ethylene; Granular cold-water-soluble starch 